\title{
Imeglimin: A Potential New Multi-Target Drug for Type 2 Diabetes
}

\author{
Valerie Vuylsteke $^{1} \cdot$ Lisa M. Chastain ${ }^{2} \cdot$ Geeta A. Maggu $^{3} \cdot$ Crystal Brown $^{2}$
}

Published online: 8 August 2015

(c) The Author(s) 2015. This article is published with open access at Springerlink.com

\begin{abstract}
Imeglimin is a novel agent currently in development to treat type 2 diabetes. Laboratory studies have demonstrated that it has the potential to impact the three main pathophysiologic components of type 2 diabetes: impaired glucose uptake by muscle tissue, excess hepatic gluconeogenesis, and increased beta-cell apoptosis. Preliminary human studies that have been published within the last 2 years demonstrate that imeglimin improves hemoglobin A1c and fasting plasma glucose similarly when compared with metformin and with sitagliptin. There has also been a low incidence of adverse effects, especially hypoglycemia, reported in these early human studies. Currently, imeglimin is lacking long-term evidence to demonstrate any effects on its cardiovascular safety, and data on morbidity and mortality, though some studies are currently in progress. There is great potential for imeglimin, if FDA approved, to play a significant role in the type 2 diabetes management algorithm.
\end{abstract}

Valerie Vuylsteke

Valerie.vuylsteke@ttuhsc.edu

1 Texas Tech School of Pharmacy-Dallas/Fort Worth Campus, 4500 S Lancaster Building 7, R\# 206, Dallas, TX 75216, USA

2 Texas Tech School of Pharmacy-Dallas/Fort Worth Campus, 4500 S. Lancaster Bldg 7, Rt \#119A, Dallas, TX 75216, USA

3 VA North Texas Medical Center-Polk Street Annex, 4243 S. Polk Street, Dallas, TX 75224, USA

\section{Key Points}

Imeglimin has been shown to have a positive impact on skeletal muscle glucose uptake, hepatic glucose production, and beta-cell apoptosis.

Imeglimin has demonstrated similar clinical benefit in hemoglobin A1c and fasting plasma glucose when compared with metformin and sitagliptin.

Although imeglimin has not yet achieved FDA approval, it is a promising new agent that could impact the treatment of type 2 diabetes.

\section{Introduction}

Type 2 diabetes (T2DM) is a widespread disease, affecting more than 380 million people worldwide, with projections that it will include more than 590 million people across the planet by 2035 [1]. To address this global concern, significant amounts of time, effort and money have been allocated to treatment, prevention and education by many entities, including private and public organizations. Despite best efforts, the prevalence of T2DM continues to rise, especially considering the increased aging population, increasing obesity rates and larger numbers of high-risk ethnic groups [2]. Additionally, the physiologic and progressive nature of diabetes requires a combination of lifestyle modifications and pharmacologic therapy in order to achieve and maintain long-term glycemic control. The primary physiologic defects of T2DM include excessive hepatic glucose production, diminished peripheral glucose uptake by insulin sensitive tissues and inadequate insulin 
secretion [3]. This article will introduce and review a new potential pharmacologic agent, imeglimin, comparing its basic and clinical activity to that of other widely used medications.

In general, pharmacologic therapy includes not only initial antihyperglycemic agents, but also more intensive approaches to glycemic control over time, often requiring multiple medications with synergistic mechanisms of action [4]. At first glance it seems that there are sufficient numbers of therapeutic classes of agents to treat T2DM, with each class varying in mechanism, safety, efficacy and route of administration; however, few drugs target all three of the main defects associated with T2DM, which is why complex medication regimens are often required to achieve glycemic control. Metformin is widely recognized as firstline therapy in multiple treatment algorithms, and is known to work primarily by limiting hepatic glucose production, one of the key defects of T2DM [3-5]. Metformin has a long track record of efficacy, minimal weight gain and low risk of hypoglycemia. Despite the potential benefits of metformin, some patients cannot tolerate the gastrointestinal (GI) side effects associated with this drug [3]. Insulin secretagogues, such as sulfonylureas and meglitinides, are also frequently used early in the treatment of a patient with diabetes as they generally elicit clinical efficacy in the short term; however, they have been noted to fail to maintain efficacy over time. While the secretagogues are targeting one of the component defects of T2DM by stimulating endogenous insulin secretion, they are often associated with hypoglycemia and weight gain, thus limiting their use. Thiazolidinediones target a third aspect of the physiologic defects of the diabetic patient by increasing insulin sensitivity in the periphery and have shown sustained durability of effect and infrequent incidence of hypoglycemia; however, they, too, have side effects that possibly limit their use, such as edema and weight gain [6].

Newer classes of antidiabetic agents that target the incretin hormones, such as glucagon-like-peptide-1 (GLP1) receptor agonists and dipeptidyl-peptidase-IV (DPP-IV) inhibitors, have recently achieved a presence in the landscape of antihyperglycemic drugs. GLP-1 receptor agonists, delivered via subcutaneous injection, promote glucose-dependent insulin secretion, inhibition of inappropriate post-prandial glucagon secretion and slowed gastric emptying to achieve glycemic control. While they are not often associated with hypoglycemia and generally induce some weight loss, the GLP-1 receptor agonists are often poorly tolerated because of nausea and increased risk of pancreatitis. Additionally, the FDA has issued a boxed warning identifying the risk of medullary thyroid cancer with the GLP-1 receptor agonists [7]. DPP-IV inhibitors also work on incretin hormones by blocking the enzyme that is responsible for degrading endogenous GLP-1, allowing for higher concentrations of GLP-1 to exert its action; however, it should be noted that glycemic effects are generally less significant with the DPP-IV inhibitors compared with the exogenous GLP-1 receptor agonists. DPP-IV inhibitors, unlike GLP-1 receptor agonists, are administered orally and generally have good patient tolerability and a low incidence of hypoglycemia; however, the overall efficacy in glucose reduction is minimal compared with other classes [8]. Selective sodium glucose cotransporter 2 (SGLT-2) inhibitors are the newest agents to gain approval for the treatment of T2DM. This class has a unique mechanism of action that does not target the pathology of diabetes, but works by increasing glucose excretion in the urine. These agents are not associated with weight gain or hypoglycemia, but are known to cause an increase in genital and urinary tract infections for both men and women [9]. Insulin therapies are by far the most efficacious agents for glycemic control and directly target insulin deficiency/insufficiency. Insulin does not have dose limitations or other side effects seen with non-insulin therapies, but does have a significant risk of hypoglycemia and weight gain, and patients are generally averse to starting insulin therapy early on in the course of their disease [3].

After reviewing these agents used for the treatment of T2DM, it is evident that no single agent targets the three primary defects of this widespread disease. Imeglimin is a drug currently in development as the first in a new class of oral antidiabetic agents acting on both insulin resistant organs and well as pancreatic beta cells. Although the precise mechanism of action has not been fully understood, it appears, based on pre-clinical results, to target several components of the pathology of T2DM. In rodent studies, it has been shown to reduce fasting plasma glucose (FPG), reduce hemoglobin A1c (A1c) and inhibit hepatic glucose production similar to metformin. Imeglimin has also been associated with stimulation of skeletal muscle glucose uptake, protection against beta-cell apoptosis, and reduction of liver steatosis. Lastly, imeglimin has been shown to perform similarly to DPP-IV inhibitors with respect to glucose-dependent insulin secretion [10]. Researchers hope this drug will add to the arsenal of existing antidiabetic agents as either an alternative or complementary option in any stage of the disease.

\section{Laboratory Studies}

Imeglimin is a novel agent unlike any other preexisting therapy for T2DM on the basis of its structure and proposed mechanism of action. Imeglimin is the first in the class of drugs called the "glimins" and, more specifically, is a tetrahydrotriazene compound [11]. Animal studies, 
primarily in rodent models, have been previously published describing the effects of imeglimin on three primary pathophysiologic defects associated with T2DM.

One such target defect of T2DM is excessive hepatic glucose production, and has been evaluated in rat hepatocytes with chemically induced T2DM [11]. Hepatocytes that were exposed to imeglimin versus placebo demonstrated a statistically significant decrease $(p<0.01)$ in the level of gluconeogenesis activity. This effect on hepatic glucose production appeared to be dosage dependent, with the highest imeglimin concentrations eliciting inhibitory activity similar to the level of efficacy of metformin, as seen in previous trials.

The second physiologic target of imeglimin is skeletal muscle glucose uptake, which has been evaluated with both in vitro and in vivo studies in rodents. In vitro studies involving isolated mouse tissue cells demonstrated a statistically significant and dose-dependent improvement $(p<0.001)$ in glucose uptake when exposed to imeglimin and glucose in their growth medium [11]. In in vivo studies, rats with chemically induced T2DM were administered oral doses of imeglimin and monitored for a 45-day treatment period. When radioactive glucose was administered to the rats, skeletal muscle uptake of these glucose molecules were monitored and recorded. Glucose uptake with all doses of imeglimin was significantly higher $(p<0.01)$ compared with controls even at the lowest dose.

The protective effect of imeglimin against beta-cell apoptosis was also tested in rats, and its effects were compared with those of exenatide, an agent known to have beta-cell protection. Isolated rat islet cells were incubated in either imeglimin or exenatide and then exposed to toxic cytokines, and the proportion of cell death was measured and compared [11]. Although no statistical significance was reported, $37 \%$ less cell apoptosis occurred in islet cells incubated in imeglimin versus $29 \%$ less cell death with exenatide exposure. These results are comparable, suggesting that imeglimin does provide some protective effects towards the beta cells of rat pancreas tissue.

Most promisingly, rat subjects with either chemically or genetically induced T2DM showed dose-dependent improvements in A1c, glucose tolerance and FPG that were comparable with metformin after a 35-day treatment with oral forms of each drug [11]. These results further support the theory that imeglimin provides similar benefit in multifaceted ways when compared with the existing treatment modalities for T2DM.

Lastly, a study of rodents fed high-fat, high-sucrose diets who received 6 weeks of imeglimin therapy also indicated significant reduction of glycemia, restoration of normal glucose tolerance, improved insulin sensitivity in liver and skeletal muscle as well as reduction of liver steatosis [12]. Unlike previous studies, however, Vial et al.
[12] found that these larger effects are likely produced by improvement in hepatic mitochondrial functioning. Vial proposed that imeglimin induces an increase in mitochondrial phospholipid composition which decreases the production of reactive oxygen species and contributes to improved functioning.

Moving forward from animal studies, four human trials have recently been published that demonstrate how imeglimin fares as monotherapy as well as adjunctive therapy with metformin or sitagliptin.

\section{Clinical Studies}

With respect to human studies, data are somewhat limited as imeglimin is still in clinical trials. Pacini et al. [13] completed a study to explore whether beta-cell function could contribute to the mechanism of action of imeglimin. In a double-blind, randomized, placebo-controlled study of 33 patients with T2DM (A1c $6.8 \pm 0.1 \%$ ) who were either treatment naïve $(n=4)$ or had only been managed with metformin $(n=29), 7$ days of imeglimin treatment significantly raised the insulin secretory response (ISR) to glucose by $112 \%(p=0.035)$, first-phase ISR by $+110 \%$ $(p=0.034)$ and second-phase ISR by $+29 \%(p=0.031)$ [13]. Imeglimin also improved beta-cell glucose sensitivity by $36 \%(p=0.034)$. The authors felt that these results were similar to preclinical studies and indicate direct effects on beta-cell function, and the mechanisms at this level require elucidation.

Pirags et al. conducted two phase II studies with different durations to (1) unravel the best drug regimen between once daily versus twice daily administration and (2) demonstrate the potential range of the active dose of imeglimin. The 4-week study was a randomized, doubleblind, double-dummy, three-arm parallel-group, multipledose study performed in Germany [11]. Fifty-nine subjects that were treatment naïve or treated with either metformin or sulfonylurea were randomized to study doses of imeglimin $2000 \mathrm{mg}$ every evening, imeglimin $1000 \mathrm{mg}$ twice daily, or metformin $850 \mathrm{mg}$ twice daily. The 4-week study demonstrated that the most notable decrease in area under the curve (AUC) plasma glucose after an oral glucose tolerance test was with imeglimin twice daily $(p<0.0001)$, followed by metformin $(p<0.0004)$ [14]. There was no significant difference between the metformin and imeglimin twice daily group $(p=0.8321)$. First-phase insulin secretion, $\mathrm{AUC}_{0-30} \mathrm{~min}$ insulin/ $\mathrm{AUC}_{0-30}$ min glucose increased with both imeglimin twice daily by $72 \%$ and metformin by $38 \%$ compared with baseline.

The second study by Pirags et al. was performed over 8 weeks as a randomized, double-blind, four-arm, controlled, multi-center study performed at seven sites in 
Latvia [11]. One hundred twenty-eight patients were randomized to imeglimin $500 \mathrm{mg}$ twice daily, imeglimin $1500 \mathrm{mg}$ twice daily, metformin $850 \mathrm{mg}$ twice daily, or placebo. This study demonstrated that imeglimin $1500 \mathrm{mg}$ twice daily, but not imeglimin $500 \mathrm{mg}$ twice daily, was significantly superior to placebo in reducing plasma glucose $\mathrm{AUC}_{0-6 \mathrm{~h}}$, but not significantly different when compared with metformin. Although not statistically significant, FPG decreased in both imeglimin $1500 \mathrm{mg}$ twice daily and metformin $850 \mathrm{mg}$ twice daily groups from baseline values, 172.26 and 169.38 to 154.26 and $144.72 \mathrm{mg} / \mathrm{dl}$, respectively [14]. The ratio of AUC insulin/ AUC glucose increased during the study in both imeglimin and metformin treatment groups compared with placebo, indicating an increase in insulin secretion.

In both studies, although the incidence of adverse events was quite different, imeglimin was generally well tolerated, with the most frequent adverse effects being headache and GI-related side effects, some of which include nausea, vomiting, and diarrhea. No serious or severe adverse effects, including echocardiographic and vital signs changes, were noted with imeglimin [14].

In a 12-week multi-center, randomized, placebo-controlled, parallel study conducted by Fouqueray et al., 156 patients were randomized $1: 1$ to receive imeglimin $1500 \mathrm{mg}$ twice daily or placebo in addition to a lead-in dose of metformin [14]. The mean metformin dose of approximately $1900 \mathrm{mg} / \mathrm{day}$ remained the same throughout the study for both treatment arms. By the completion of the study, A1c decreased by $0.65 \%$ in the imeglimin group and by $0.21 \%$ in the placebo group $(p=0.001)$ from a baseline A1c of 8.5 and $8.6 \%$ in the imeglimin and placebo arms, respectively [14]. Additionally, there were statistically significant reductions in FPG $(p<0.001)$ and the proinsulin/insulin ratio $(p=0.007)$ from baseline compared with placebo. Greater than $60 \%$ of patients in the imeglimin group achieved a decrease in A1c of $\geq 0.5 \%$ compared with $36.4 \%$ in the placebo group. Around $14 \%$ of subjects receiving imeglimin achieved an A1c of $<7 \%$ compared with only $3.8 \%$ of participants in the placebo group.

Overall, imeglimin was well tolerated without any serious cardiovascular events or adverse events. Of note, there were no statistically significant changes in mean body weight $(p=0.08)$ and waist circumference $(p=0.053)$ in the imeglimin versus placebo group [14]. This study demonstrated that in combination with metformin, there were clinically and statistically significant reductions in the A1c, FPG, and proinsulin/insulin ratio. Additionally, this novel drug in a class of its own can be offered as an add-on therapy with metformin as a potential alternative to other second-line agents.

Fouqueray et al. also assessed the safety and efficacy of add-on imeglimin therapy in patients inadequately controlled on sitagliptin monotherapy in a 12-week, 29-center, three-country, randomized, double-blind, placebo-controlled, parallel study of 170 subjects with T2DM [15]. The researchers conducted a 12-week run-in period in which all subjects were converted from current therapy (10\% were treatment naïve vs. a $90 \%$ majority on metformin therapy) to sitagliptin $100 \mathrm{mg} /$ day for 10 weeks, followed by 2 weeks of sitagliptin and placebo. Baseline labs were drawn at the end of this 12 -week run-in period, and patients were then randomized to receive sitagliptin $100 \mathrm{mg} /$ day plus imeglimin $1500 \mathrm{mg}$ twice daily or placebo. Monitoring was carried out at baseline and at weeks 4 , 8 and 12 . There was a statistically significant difference in change in A1c from baseline (baseline A1c of $8.5 \%$ ) in the sitagliptin + imeglimin group $(-0.6 \%)$ compared with the sitagliptin + placebo group $(+0.12 \%)(-0.72 \%$ difference; $p<0.001)$ and in FPG $(-16.75 \mathrm{mg} / \mathrm{dl}$ vs. $-1.98 \mathrm{mg} /$ $\mathrm{dl} ;-14.77 \mathrm{mg} / \mathrm{dl}$ difference; $p=0.014)$. Larger reductions in A1c were seen in patients with higher baseline A1c ($0.9 \%$ with baseline A1c of $>9 \%$ ). There was no significant difference between the groups with regard to beta-cell function parameters, as was expected: insulin (uIU/ml), c-peptide $(\mathrm{ng} / \mathrm{ml})$, proinsulin/insulin ratio and/or homeostatic model assessment of insulin resistance. No significant differences from baseline to week 12 between groups were identified with respect to triglycerides, C-reactive protein or systolic blood pressure. Weight and waist circumference were not impacted in the combination group compared with sitagliptin alone as seen in prior studies (reduced parameters) [11]. There were no reported hypoglycemic events reported in the combination group. Additionally, there were fewer adverse events in the imeglimin group versus the placebo group [seven events in three subjects (one requiring rescue therapy) in placebo vs. none in the imeglimin group] [15]. As in the previously mentioned study with metformin, this study demonstrated that imeglimin is well tolerated and efficacious in reducing A1c when added to sitagliptin monotherapy, making it a potential complement to either metformin or sitagliptin for the treatment of T2DM. See Table 1 for a summary of study evidence.

\section{Discussion and Conclusions}

Despite all of the promising preliminary information currently available, further evidence is needed to support the long-term safety of imeglimin and ultimately result in its FDA approval. Currently, there is one known study involving imeglimin that has been completed, but its results are yet to be published. This study is a randomized, prospective, placebo-controlled trial based in Latvia that intends to assess both efficacy and safety endpoints with 24 weeks of imeglimin treatment [16]. Patients 
Table 1 Summary of clinical study evidence

\begin{tabular}{|c|c|c|c|c|}
\hline References & Imeglimin dose & Comparator & Results & $P$ value \\
\hline \multirow{2}{*}{$\begin{array}{l}\text { Fouqueray } \\
\text { et al. [15] }\end{array}$} & \multirow{2}{*}{$\begin{array}{l}1500 \mathrm{mg} \text { BID + } \\
\text { sitagliptin }\end{array}$} & \multirow[t]{2}{*}{ Placebo + sitagliptin } & Change in A1c from baseline at 12 weeks $(-0.6$ vs. $+0.12 \%)$ & $<0.001$ \\
\hline & & & $\begin{array}{l}\text { Change in FPG from baseline at } 12 \text { weeks }(-16.75 \\
\text { vs. }-1.98 \mathrm{mg} / \mathrm{dl})\end{array}$ & 0.014 \\
\hline \multirow{3}{*}{$\begin{array}{l}\text { Fouqueray } \\
\text { et al. [14] }\end{array}$} & \multirow{3}{*}{$\begin{array}{l}1500 \mathrm{mg} \text { BID }+ \\
\text { metformin }\end{array}$} & \multirow[t]{3}{*}{ Placebo + metformin } & Change in A1c from baseline at 12 weeks ( -0.65 vs. $-0.21 \%)$ & $<0.001$ \\
\hline & & & $\begin{array}{l}\text { Change in FPG from baseline at } 12 \text { weeks }(-0.91 \mathrm{mg} / \mathrm{dl} \\
\text { vs. }+0.36 \mathrm{mg} / \mathrm{dl})\end{array}$ & $<0.001$ \\
\hline & & & $\begin{array}{l}\text { Change from baseline in proinsulin/insulin ratio at week } 12 \\
(-7.5 \text { vs. }+11.81)\end{array}$ & $<0.007$ \\
\hline \multirow[t]{6}{*}{$\begin{array}{l}\text { Pirags et al. } \\
\text { [11] }\end{array}$} & $2000 \mathrm{mg}$ once daily & \multirow[t]{2}{*}{$\begin{array}{l}\text { Metformin } 850 \mathrm{mg} \\
\text { twice daily }\end{array}$} & $\begin{array}{l}\text { Change in baseline of AUC plasma glucose at } 4 \text { weeks } \\
(-10 \text { vs. } 30 \%)\end{array}$ & $\begin{array}{l}<0.0001 \\
<0.0004\end{array}$ \\
\hline & $1000 \mathrm{mg}$ twice daily & & $\begin{array}{l}\text { Change in baseline of AUC plasma glucose at } 4 \text { weeks } \\
(-33 \text { vs. } 30 \%)\end{array}$ & $\begin{array}{l}<0.0305 \\
<0.0004\end{array}$ \\
\hline & $500 \mathrm{mg}$ twice daily & \multirow[t]{4}{*}{$\begin{array}{l}\text { Metformin } 850 \mathrm{mg} \\
\text { twice daily or } \\
\text { placebo }\end{array}$} & $\begin{array}{l}\text { AUC glucose at week } 8(72,661 \text { vs. } 58,054 \text { or } 78,174 \mathrm{mg} / \mathrm{dl}) \\
\text { FPG at week } 8(172 \text { vs. } 144.7 \text { or } 182 \mathrm{mg} / \mathrm{dl}) \\
\text { Alc at week } 8(7.58 \text { vs. } 6.97 \text { or } 7.52 \%)\end{array}$ & \multirow[t]{4}{*}{$\begin{array}{l}\text { Not } \\
\text { provided }\end{array}$} \\
\hline & \multirow[t]{3}{*}{$1500 \mathrm{mg}$ twice daily } & & AUC glucose at week 8 (63,293 vs. 58,054 or $78,174 \mathrm{mg} / \mathrm{dl})$ & \\
\hline & & & FPG at week 8 (154 vs. 144.7 or $182 \mathrm{mg} / \mathrm{dl})$ & \\
\hline & & & A1c at week 8 ( 7.17 vs. 6.97 or $7.52 \%)$ & \\
\hline
\end{tabular}

$A U C$ area under the curve, $A 1 c$ hemoglobin A1c, BID twice a day, $F P G$ fasting plasma glucose

included in this trial may be either treatment naïve or have been previously treated with oral monotherapy. Trials assessing the safety and efficacy of imeglimin with insulin therapy or compared directly with insulin are yet to be published.

Ultimately, the place that imeglimin will have in the current T2DM treatment algorithm is unknown. At this time, there are no available data that investigate the impact of imeglimin on morbidity and/or mortality in patients with T2DM. More data that support the long-term safety of imeglimin are necessary as well. However, based on the results of the animal studies and human trials that have been published recently, imeglimin seems to be a promising adjunct, or even a potential initial monotherapy agent, in treating T2DM.

Acknowledgments The authors would like to acknowledge each other in the preparation and review of this manuscript.

\section{Compliance with Ethical Standards}

Funding No funding was received for the preparation of this manuscript.

Conflicts of interest The authors, Valerie Vuylsteke, Lisa M. Chastain, Geeta A. Maggu and Crystal Brown, disclose that they have no conflicts of interest regarding the outcomes of the research presented and reviewed in this manuscript.

Open Access This article is distributed under the terms of the Creative Commons Attribution-NonCommercial 4.0 International License (http://creativecommons.org/licenses/by-nc/4.0/), which permits any noncommercial use, distribution, and reproduction in any medium, provided you give appropriate credit to the original author(s) and the source, provide a link to the Creative Commons license, and indicate if changes were made.

\section{References}

1. International Diabetes Foundation. Diabetes: facts and figures. Available from: http://www.idf.org/worlddiabetesday/toolkit/gp/ facts-figures. Cited 16 Nov 2013.

2. DeFronzo RA, Bonadonna RC, Ferrannini E. Pathogenesis of NIDDM. A balanced overview. Diabetes Care. 1992;15:318-68.

3. Inzucchi SE, Bergenstal RM, Buse JB, et al. Management of hyperglycemia in type 2 diabetes: a patient-centered approach: position statement of the American Diabetes Association (ADA) and the European Association for the Study of Diabetes (EASD). Diabetes Care. 2012;35(6):1334-79.

4. Garber AJ, Abrahamson MJ, Barzilay JI, et al. AACE comprehensive diabetes management algorithm 2013 consensus statement. Endocr Pract. 2013;19(Suppl 2):1-48.

5. Qaseem A, Humphrey L, Sweet D, et al. Oral pharmacologic treatment of type 2 diabetes mellitus: a clinical practice guideline from the American College of Physicians. Ann Intern Med. 2012;156:218-31.

6. Actos [package insert]. Deerfield: Takeda Pharmaceutical; 2013.

7. Byetta [package insert]. San Diego: Amylin Pharmaceutical; 2011.

8. Januvia [package insert]. Whitehouse Station: Merck \& Co; 2014.

9. Farxiga [package insert]. Princeton: Bristol-Myers Squibb; 2014.

10. Fouqueray $P$, Leverve $X$, Fontaine E, et al. Imeglimin-a new oral anti-diabetic that targets the three key defects of type 2 diabetes. J Diabetes Metab. 2011;2:126.

11. Pirags V, Lebovitz H, Fouqueray P. Imeglimin, a novel oral antidiabetic, exhibits a good efficacy and safety profile in type 2 diabetic patients. Diabetes Obes Metab. 2012;14:852-8. 
12. Vial G, Chauvin MA, Bendridi N, Durand A, Meugnier E, Madec AM, Bernoud-Hubac N, Pais de Barros JP, Fontaine É, Acquaviva C, Hallakou-Bozec S, Bolze S, Vidal H, Rieusset J. Imeglimin normalizes glucose tolerance and insulin sensitivity and improves mitochondrial function in liver of a high-fat, high-sucrose diet mice model. Diabetes. 2015;64(6):2254-64.

13. Pacini G, Mari A, Fouqueray P, Bolze S, Roden M. Imeglimin increases glucose-dependent insulin secretion and improves betacell function in patients with type 2 diabetes. Diabetes Obes Metab. 2015;17:541-5.

14. Fouqueray P, Pirags V, Inzucchi SE, Bailey CJ, Schernthaner G, Diamant $\mathrm{M}$, et al. The efficacy and safety of imeglimin as add-on therapy in patients with type 2 diabetes inadequately controlled with metformin monotherapy. Diabetes Care. 2013;36(3):565-8.

15. Fouqueray P, Pirags V, Diamant M, Schernthaner G, Lebovitz HE, Inzucchi SE, et al. The efficacy and safety of imeglimin as add-on therapy in patients with type 2 diabetes inadequately controlled with sitagliptin monotherapy. Diabetes Care. 2014;37(7):1924-30.

16. A study of the efficacy and safety of 4 doses of imeglimin after 24 weeks of treatment in subjects with type 2 diabetes. http:// www.clinicaltrials.gov. Accessed 1 Jun 2015. 